\title{
Pressure balance and imbalance in the optic nerve chamber: The Beijing Intracranial and Intraocular Pressure (iCOP) Study
}

\author{
Ruowu Hou ${ }^{1 \dagger}$, Zheng Zhang ${ }^{2 \dagger}$, Diya Yang ${ }^{2,3}$, Huaizhou Wang ${ }^{2,3}$, Weiwei Chen ${ }^{3}$, Zhen $\mathrm{Li}^{4}$, \\ Jinghong Sang ${ }^{2}$, Sumeng $\mathrm{Liu}^{2}$, Yiwen $\mathrm{CaO}^{2}$, Xiaobin Xie ${ }^{5}$, Ruojing Ren ${ }^{6}$, Yazhuo Zhang ${ }^{7 *}$, \\ Bernhard A. Sabel ${ }^{8}$ \& Ningli Wang ${ }^{2,3 \ddagger}$ \\ ${ }^{1}$ Department of Neurosurgery, Beijing Tongren Hospital, Capital Medical University, Beijing 100730, China; \\ ${ }^{2}$ Beijing Tongren Eye Center, Beijing Tongren Hospital, Capital Medical University, Beijing Ophthalmology and Visual Sciences Key \\ Laboratory, Beijing 100730, China; \\ ${ }^{3}$ Beijing Institute of Ophthalmology, Beijing Tongren Hospital, Capital Medical University, Beijing 100730, China; \\ ${ }^{4}$ Department of Ophthalmology, Xuanwu Hospital, Capital Medical University, Beijing 100053, China; \\ ${ }^{5}$ Department of Ophthalmology, Eye Hospital of China Academy of Chinese Medical Sciences, Beijing 100040, China; \\ ${ }^{6}$ Eye center, New York Eye and Ear Infirmary, New York 10003, USA \\ ${ }^{7}$ Department of Endoscopic and Microinvasive Neurosurgery, Beijing Neurosurgery Institute, Beijing Tiantian Hospital, Capital Medical \\ University, Beijing 100050, China; \\ ${ }^{8}$ Institute of Medical Psychology, Medical Faculty, Otto-v.-Guericke University of Magdeburg, Magdeburg 39120, Germany
}

Received December 27, 2015; accepted January 25, 2016; published online February 25, 2016

\begin{abstract}
To determine the interdependence of intracranial pressure (ICP) and intraocular pressure (IOP) and how it affects optic nerve pressures, eight normal dogs were examined using pressure-sensing probes implanted into the left ventricle, lumbar cistern, optic nerve subarachnoid space in the left eye, and anterior chamber in the left eye. This allowed ICP, lumbar cistern pressure (LCP), optic nerve subarachnoid space pressure (ONSP) and IOP to be simultaneously recorded. After establishing baseline pressure levels, pressure changes that resulted from lowering ICP (via shunting cerebrospinal fluid (CSF) from the ventricle) were recorded. At baseline, all examined pressures were different $(\mathrm{ICP}>\mathrm{LCP}>\mathrm{ONSP})$, but correlated $(P<0.001)$. As ICP was lowered during CSF shunting, IOP also dropped in a parallel time course so that the trans-lamina cribrosa gradient (TLPG) remained stable (ICP-IOP dependent zone). However, once ICP fell below a critical breakpoint, ICP and IOP became uncoupled and TLPG changed as ICP declined (ICP-IOP independent zone). The optic nerve pressure gradient (ONPG) and trans-optic nerve pressure gradient (TOPG) increased linearly as ICP decreased through both the ICP-IOP dependent and independent zones. We conclude that ICP and IOP are coupled in a specific pressure range, but when ICP drops below a critical point, IOP and ICP become uncoupled and TLPG increases. When ICP drops, a rise in the ONPG and TOPG creates more pressure and reduces CSF flow around the optic nerve. This change may play a role in the development and progression of various ophthalmic and neurological diseases, including glaucoma.
\end{abstract}

glaucoma, optic neuropathy, trans-lamina cribrosa pressure gradient (TLPG), trans-optic canal pressure gradient (TCPG), trans-optic nerve pressure gradient (TOPG), optic nerve pressure gradient (ONPG)

Citation: Hou, R., Zhang, Z., Yang, D., Wang, H., Chen, W., Li, Z., Sang, J., Liu, S., Cao, Y., Xie, X., Ren, R., Zhang, Y., Sabel, B.A., and Wang, N. (2016). Pressure balance and imbalance in the optic nerve chamber: The Beijing Intracranial and Intraocular Pressure (iCOP) Study. Sci China Life Sci 59, 495-503. doi: 10.1007/s11427-016-5022-9

$\dagger$ Contributed equally to this work

*Corresponding author (email: zyz2004520@yeah.net)

†Corresponding author (email: wningli@vip.163.com) 


\section{INTRODUCTION}

Glaucomatous optic neuropathy that damages the retina or optic nerve may be a pathological response to altered physiological pressure gradients created by both intraocular pressure (IOP) and intracranial pressure (ICP). Traditionally, IOP is thought to primarily influence the optic nerve and that IOP elevation is the key issue in glaucoma. However, Berdahl et al. suggested that ICP might also be a critical factor for determining pressure on the optic nerve (Berdahl et al., 2008a, b; Ren et al., 2010). This theory is compatible with the experimental observation that lowering cerebrospinal fluid (CSF) pressure over the course of one year, via CSF shunting from the lumbar cistern to the abdomen, can lead to optic nerve damage in monkeys (Yang et al., 2014). Therefore, it is possible that glaucoma may be caused by high IOP, low ICP, or a combination of both.

Exogenous pressure changes, other than endogenous pressure changes found in glaucoma, can also affect the optic nerve. For example, long-term exposure to a microgravity environment may simultaneously elevate ICP and IOP and cause visual impairment (Mader et al., 2011; Woo et al., 2012). During this condition, ICP and IOP are altered together. Moreover, Wostyn et al. argued that Alzheimer's disease (AD) shares some features with glaucoma in that both conditions lead to retinal ganglion cell death (Wostyn et al., 2008, 2010). Indeed, there is a higher incidence of normal tension glaucoma in patients with AD (Bach-Holm et al., 2012) and this link may provide some clues to possible links between ICP and IOP. Figure 1 summarizes some diseases associated with ICP and IOP, some of which are not traditionally regarded as separate illnesses. However, examining ICP and IOP status separately reveals that some conditions may very well be distinct.

It is known that ICP and IOP influence optic nerve pressure, but it is not known how these two pressures are related. Here, we examine ICP and IOP in healthy dogs with acutely lowered ICP (via CSF shunting) to explore the relationship of the pressure conditions in the eye and the three different CSF cavities (optic nerve subarachnoid space (SAS), ventricle, and lumbar cistern).

\section{RESULTS}

Average baseline IOP, ICP, Lumbar cistern pressure (LCP), and optic nerve SAS pressure (ONSP) was 181.4 \pm 11.4 , $105.3 \pm 14.1,87.9 \pm 14.3$, and $59.3 \pm 8.7 \mathrm{mmH}_{2} \mathrm{O}$, respectively (Table 1). Thus, ONSP was only about $60 \%$ of ICP. The IOP was significantly higher than ICP $(P<0.001)$, ICP was significantly higher than both LCP $(P<0.001)$ and ONSP $(P<0.001)$, and LCP was significantly higher than ONSP $(P<0.001)$.

Figure 2 shows the relationship between ICP and IOP and clearly illustrates three definable parameters, the ICP-

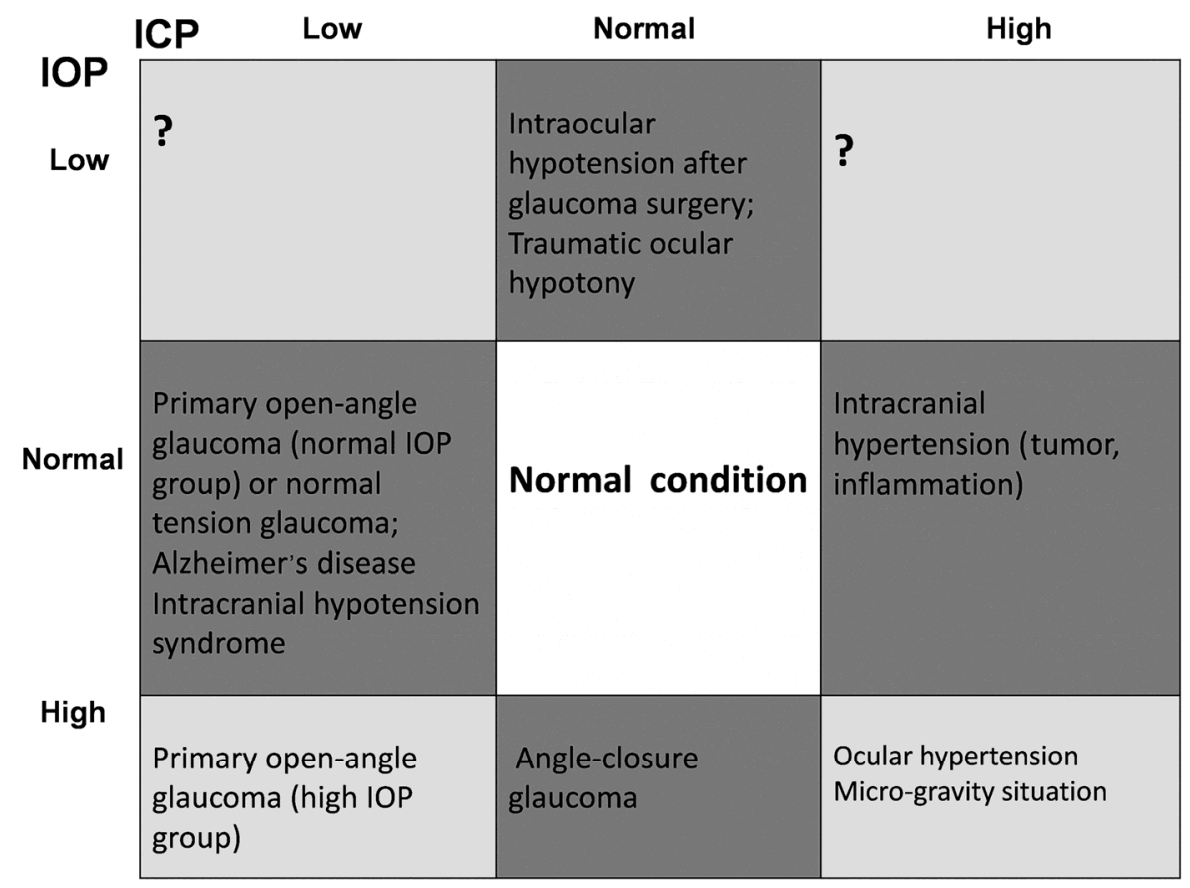

Figure 1 Diseases associated with intracranial pressure (ICP) and intraocular pressure (IOP). Please note that a low-ICP/low-IOP condition is rare in the clinical situation because subjects generally do not complain of symptoms. In patients with high-ICP/low-IOP patients, symptoms of high ICP are reported and generally attended to by a neurologist, who typically does not assess IOP. Similarly, in primary open-angle glaucoma (high IOP group), ophthalmologists focus on the high IOP and dismiss the status of ICP, which is also difficult to measure in the clinic. A micro-gravity situation is rare on Earth and patients with ocular hypertension, in general, do not complain of visual dysfunction. The other conditions are more common in the clinic and these have been sorted based on hypothesis. 
Table 1 Normal intracranial pressure (ICP), lumbar cistern pressure (LCP), optic nerve subarachnoid space pressure (ONSP), intraocular pressure (IOP), optic nerve pressure gradient (ONPG), trans-optic nerve pressure gradient (TOPG), and trans-lamina cribrosa pressure gradient (TLPG) in all study dogs $\left(\mathrm{mmH}_{2} \mathrm{O}\right)$. Data are presented as mean \pm standard deviation

\begin{tabular}{|c|c|c|c|c|c|c|c|}
\hline \multirow{2}{*}{ Animal } & \multirow{2}{*}{$\mathrm{ICP}$} & \multirow{2}{*}{$\mathrm{LCP}$} & \multirow{2}{*}{ ONSP } & \multirow{2}{*}{ IOP } & ONPG & TOPG & TLPG \\
\hline & & & & & IOP-ICP & ONPG-ONSP & IOP-ONSP \\
\hline 1 & $120.7 \pm 5.0$ & $110.0 \pm 6.6$ & $70.9 \pm 6.3$ & $190.2 \pm 1.2$ & $69.5 \pm 4.6$ & $-1.5 \pm 9.2$ & $119.2 \pm 5.4$ \\
\hline 2 & $85.2 \pm 1.7$ & $71.3 \pm 1.9$ & $45.8 \pm 1.9$ & $163.4 \pm 5.4$ & $76.1 \pm 6.2$ & $30.3 \pm 7.3$ & $117.5 \pm 6.4$ \\
\hline 3 & $95.2 \pm 2.1$ & $76.2 \pm 1.6$ & $54.9 \pm 2.9$ & $174.7 \pm 2.4$ & $79.5 \pm 4.0$ & $24.5 \pm 6.8$ & $119.7 \pm 5.2$ \\
\hline 4 & $108.9 \pm 4.0$ & $92.1 \pm 4.4$ & $49.3 \pm 3.4$ & $185.5 \pm 3.9$ & $76.6 \pm 6.0$ & $27.2 \pm 4.2$ & $136.2 \pm 0.6$ \\
\hline 5 & $93.2 \pm 2.7$ & $71.4 \pm 3.1$ & $63.3 \pm 2.5$ & $167.8 \pm 3.4$ & $74.6 \pm 4.4$ & $11.3 \pm 3.0$ & $104.6 \pm 1.8$ \\
\hline 6 & $123.0 \pm 5.6$ & $93.8 \pm 4.6$ & $67.4 \pm 1.4$ & $185.6 \pm 0.5$ & $62.6 \pm 5.4$ & $-4.8 \pm 5.9$ & $118.2 \pm 1.6$ \\
\hline 7 & $117.2 \pm 4.5$ & $102.4 \pm 5.9$ & $62.6 \pm 2.7$ & $195.6 \pm 0.9$ & $78.4 \pm 4.9$ & $15.8 \pm 2.6$ & $133.0 \pm 2.1$ \\
\hline 8 & $99.1 \pm 5.5$ & $85.8 \pm 4.4$ & $60.6 \pm 2.0$ & $188.4 \pm 0.7$ & $89.3 \pm 5.4$ & $28.7 \pm 6.1$ & $127.8 \pm 2.5$ \\
\hline Mean & $105.3 \pm 14.1$ & $87.9 \pm 14.3$ & $59.3 \pm 8.7$ & $181.4 \pm 11.4$ & $75.8 \pm 7.7$ & $16.4 \pm 13.7$ & $122.0 \pm 10.0$ \\
\hline Percent ICP (\%) & 100.0 & 83.5 & 56.3 & 172.3 & 72.0 & 15.5 & 115.9 \\
\hline
\end{tabular}
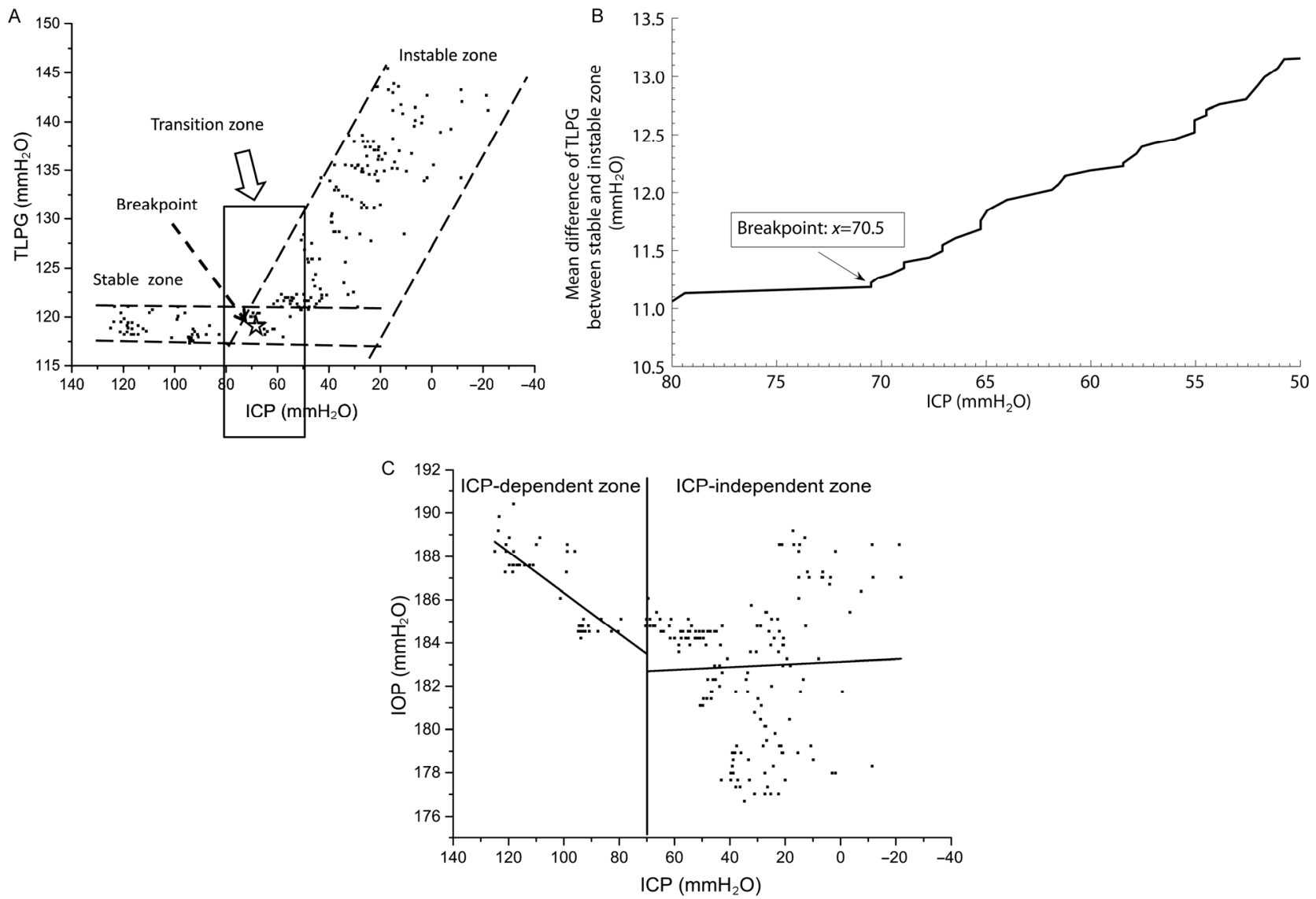

Figure 2 Correlation between intracranial pressure (ICP) and intraocular pressure (IOP). A, ICP-TLPG plot of a single dog subdivided into three parts from left to right as a function of decreasing ICP. In the stable zone TLPG remains stable despite increasing ICP. In the instable zone at lower ICP levels the TLPG rises. In the transition region, where both zones overlap, there is a "breakpoint" which indicates the critical ICP below which the TLPG starts rising. To calculate the true ICP breakpoint value, which could somewhere between 50 to $80 \mathrm{mmH}_{2} \mathrm{O}$, we applied the following methodology: we set an "arbitrary" breakpoint value inside the transition zone which best approximates where the TLPG rise begins. Next, we calculate the average and the variability band (two standard deviations) of all values to the left of the "arbitrary breakpoint". The "true breakpoint" is the ICP level below which the TLPG values are first found outside the variability band. To obtain a single line plot for this dog, we calculated the difference between the average ICP values in the stable zone and the instable zone as a function of the respective ICP level. As can be seen in (B), the line is flat until reaching an ICP of 70.5 mmH ${ }_{2} \mathrm{O}$ (the breakpoint) and rises as the ICP declines further; it is the point where the TLPG starts to rise. C, Relationship between ICP and IOP in one dog as a function of declining ICP. The curve in the "ICP-dependent zone," where IOP decreases linearly with the ICP (regression equation: $y=-0.11 x+175.1 ; r=0.832$ ), and the curve in the "ICP-independent zone", where IOP and ICP are uncoupled ( $y=-0.003 x+180.0 ; r=-0.016)$. The breakpoint (B) between the dependent and independent zones is at approximately $70 \mathrm{mmH}_{2} \mathrm{O}$. 
IOP dependent zone, the breakpoint, and the ICP-IOP independent zone. In the ICP-IOP dependent zone, IOP and ICP decrease together and the trans-lamina cribrosa pressure gradient (TLPG) was relatively stable. However, once the breakpoint had been reached and IOP and ICP are no longer synchronous (ICP-IOP independent zone), the decline in TLPG varied with the decline in ICP. For example, in one dog (Figure 2C), as long as ICP remained above 70 $\mathrm{mmH}_{2} \mathrm{O}$, IOP dropped synchronously with ICP (regression equation: $y=-0.11 x+175.1, r=0.832$ ). However, below this breakpoint of $70 \mathrm{mmH}_{2} \mathrm{O}$, IOP was not affected by further ICP decline $(y=-0.003 x+180.0 ; r=-0.016)$. A paired $t$-test revealed that average TLPG in the ICP-IOP dependent zone was not statistically different from that measured at baseline $(P=0.680)$. Interestingly, the breakpoint had considerable variation between dogs, ranging between $56.9 \%$ and $80.7 \%$ of ICP baseline values. The average breakpoint for all dogs was found to be approximately $64 \%$ (Table 2). Therefore, we normalized data used in-group analyses by defining baseline ICP of each dog as $100 \%$. The percent change in ICP over baseline (ICP\%) that occurred during CSF drainage was then calculated. The relative percentage change in IOP compared to baseline (IOP\%) was also calculated using the same method, and the ICP-IOP curve was displayed as the change of $\mathrm{ICP} \%-\mathrm{IOP} \%$. The two separate regression lines were again calculated after data were split at the breakpoint (about 64\%) between the ICP-IOP dependent and ICP-IOP independent zones. When data from all animals were examined together, the ICP\%-IOP\% regression lines were $y=-0.08 x+91.60(r=0.826)$ in the ICP-IOP dependent zone and $y=0.0006 x+96.19 \quad(r=0.007)$ in the ICP-IOP independent zone.

Table 3 shows that the trans-optic nerve pressure gradient (TOPG) was $16.4 \pm 13.7 \mathrm{mmH}_{2} \mathrm{O}$ at baseline, $38.8 \pm 15.1$ $\mathrm{mmH}_{2} \mathrm{O}$ in the ICP-IOP dependent zone and 92.8 \pm 13.2 $\mathrm{mmH}_{2} \mathrm{O}$ in the ICP-IOP independent zone. As shown in Figure 3, the optic nerve pressure gradient (ONPG) was negatively and linearly correlated with ICP.

Table 2 Trans-lamina cribrosa pressure gradient (TLPG) at baseline and in the zone where intracranial pressure (ICP) and intraocular pressure (IOP) are coupled (ICP-IOP dependent zone). Data are also shown at the breakpoint and in the ICP-IOP independent zone. Data are presented as mean \pm SD

\begin{tabular}{|c|c|c|c|c|c|}
\hline \multirow[b]{2}{*}{ Animal } & \multicolumn{5}{|c|}{ TLPG=IOP-ONSP } \\
\hline & $\begin{array}{l}\text { Baseline } \\
\left(\mathrm{mmH}_{2} \mathrm{O}\right)\end{array}$ & $\begin{array}{l}\text { ICP-IOP dependent zone } \\
\left(\mathrm{mmH}_{2} \mathrm{O}\right)\end{array}$ & $\begin{array}{l}\text { Breakpoint (ICP) } \\
\left(\mathrm{mmH}_{2} \mathrm{O}\right)\end{array}$ & $\begin{array}{c}\text { Breakpoint (Percent ICP) } \\
\%\end{array}$ & $\begin{array}{l}\text { ICP-IOP independent zone } \\
\left(\mathrm{mmH}_{2} \mathrm{O}\right)\end{array}$ \\
\hline 1 & $119.2 \pm 5.4$ & $118.7 \pm 1.0$ & 70 & 58.00 & $130.0 \pm 7.9$ \\
\hline 2 & $117.5 \pm 6.4$ & $112.9 \pm 9.3$ & 65 & 76.29 & $137.3 \pm 10.0$ \\
\hline 3 & $119.7 \pm 5.2$ & $110.9 \pm 1.0$ & 55 & 57.77 & $127.6 \pm 9.7$ \\
\hline 4 & $136.2 \pm 0.6$ & $129.7 \pm 1.6$ & 66 & 60.61 & $130.8 \pm 5.8$ \\
\hline 5 & $104.6 \pm 1.8$ & $109.5 \pm 5.5$ & 56 & 60.09 & $115.0 \pm 12.9$ \\
\hline 6 & $118.2 \pm 1.6$ & $137.3 \pm 0.9$ & 70 & 56.91 & $139.8 \pm 2.0$ \\
\hline 7 & $133.0 \pm 2.1$ & $136.7 \pm 4.5$ & 80 & 68.26 & $155.5 \pm 3.7$ \\
\hline 8 & $127.8 \pm 2.5$ & $131.2 \pm 2.5$ & 80 & 80.73 & $147.4 \pm 8.3$ \\
\hline Mean & $122.0 \pm 10.0$ & $123.4 \pm 11.7$ & $67.8 \pm 9.4$ & $64.83 \pm 9.2$ & $135.4 \pm 12.5$ \\
\hline
\end{tabular}

a) Statistical significance was examined using paired $t$-tests. There was no significant difference between the baseline and the ICP-IOP dependent zone $(P=0.680)$. However, there was a significant difference between the ICP-IOP independent zone and the baseline $(P=0.005)$. The difference between the ICP-IOP independent and ICP-IOP independent zones was also statistically significant $(P=0.005)$.

Table 3 Trans-optic nerve pressure gradient (TOPG) at baseline, in the intracranial (ICP)-intraocular pressure (IOP) dependent zone, and in the ICP-IOP independent zone a) $^{2}$

\begin{tabular}{|c|c|c|c|c|c|}
\hline \multirow[b]{2}{*}{ Animal } & \multicolumn{5}{|c|}{ TOPG $=$ ONPG $-\mathrm{ONSP}$} \\
\hline & $\begin{array}{l}\text { Baseline } \\
\left(\mathrm{mmH}_{2} \mathrm{O}\right)\end{array}$ & $\begin{array}{l}\text { ICP-IOP dependent zone } \\
\left(\mathrm{mmH}_{2} \mathrm{O}\right)\end{array}$ & $\begin{array}{l}\text { Breakpoint (ICP) } \\
\left(\mathrm{mmH}_{2} \mathrm{O}\right)\end{array}$ & $\begin{array}{c}\text { Breakpoint (Percent ICP) } \\
\%\end{array}$ & $\begin{array}{l}\text { ICP-IOP independent zone } \\
\left(\mathrm{mmH}_{2} \mathrm{O}\right)\end{array}$ \\
\hline 1 & $-1.5 \pm 9.2$ & $14.4 \pm 13.1$ & 70 & 58.00 & $95.9 \pm 27.5$ \\
\hline 2 & $30.3 \pm 7.3$ & $38.5 \pm 12.5$ & 65 & 76.29 & $84.2 \pm 22.0$ \\
\hline 3 & $24.5 \pm 6.8$ & $51.2 \pm 3.9$ & 55 & 57.77 & $82.8 \pm 23.1$ \\
\hline 4 & $27.2 \pm 4.2$ & $49.5 \pm 8.9$ & 66 & 60.61 & $112.1 \pm 16.9$ \\
\hline 6 & $-4.8 \pm 5.9$ & $58.9 \pm 4.7$ & 70 & 56.91 & $88.3 \pm 14.1$ \\
\hline 7 & $15.8 \pm 2.6$ & $41.8 \pm 13.3$ & 80 & 68.26 & $109.3 \pm 21.9$ \\
\hline 8 & $28.7 \pm 6.1$ & $34.7 \pm 11.8$ & 80 & 80.73 & $96.3 \pm 25.5$ \\
\hline Mean & $16.4 \pm 13.7$ & $38.8 \pm 15.1$ & $67.8 \pm 9.4$ & $64.83 \pm 9.2$ & $92.8 \pm 13.2$ \\
\hline
\end{tabular}

a) Paired $t$-tests were used to determine statistical significance of differences between the baseline and the ICP-IOP dependent zone $(P=0.011)$. The difference between the ICP-IOP independent zone and the baseline was also significant $(P<0.001)$. Likewise, the difference between the ICP-IOP dependent and independent zones was significant $(P<0.001)$. 

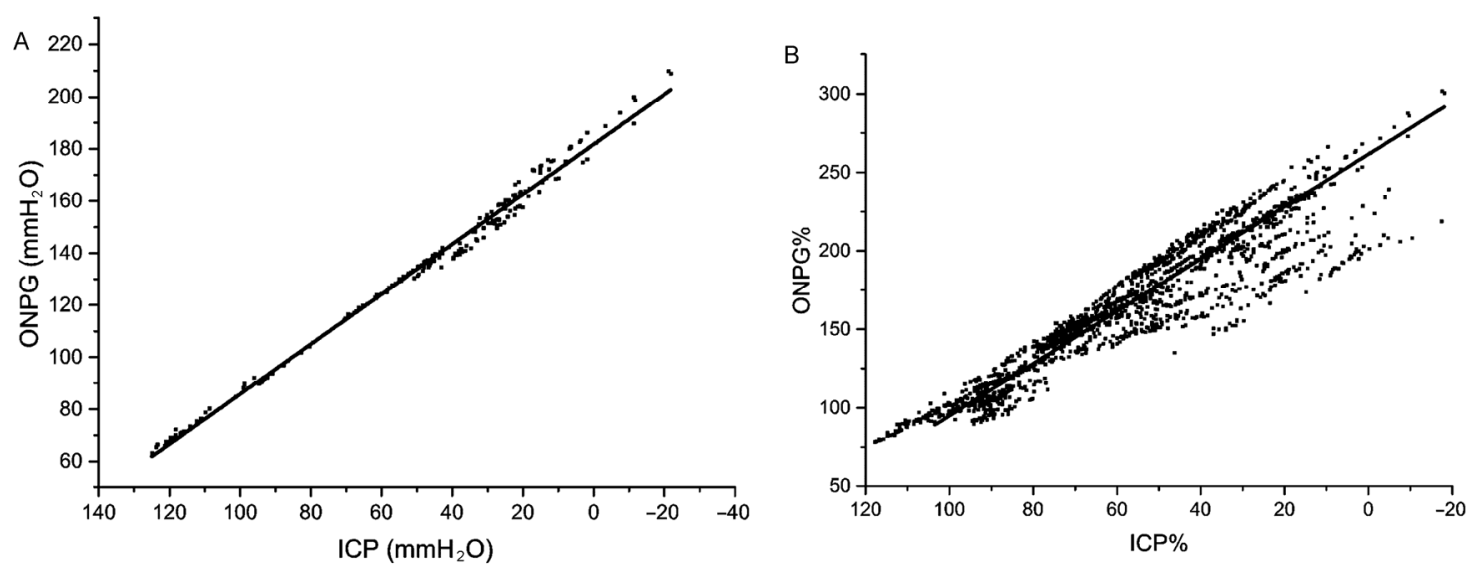

Figure 3 The relationship between intracranial pressure (ICP) and the optic nerve pressure gradient (ONPG). A, Relationship between ICP and ONPG in one dog as a function of declining ICP. The ONPG was negatively and linearly correlated with ICP (regression equation: $y=-0.96 x+181.8$; $r=-0.996$ ). B, The ICP\%-ONSP\% curve for all animals combined. Regression lines are shown $(y=-1.67 x+261.56, r=-0.996)$. Calculations are described in the methods section of the main text.

\section{DISCUSSION}

By analyzing the correlation between ICP and IOP in eight normal dogs, we are the first to identify three different features of the ICP-IOP relationship, including an ICP-IOP dependent zone, a breakpoint, and an ICP-IOP independent zone. This shows that the relationship between ICP and IOP is not always the same. Namely, IOP and ICP are coupled in the ICP-IOP dependent zone (i.e., changes occur in parallel leaving TLPG unchanged). However, there is also an ICP-IOP independent zone, which begins when ICP levels are at or below a critical breakpoint, in which ICP and IOP become uncoupled. In this zone, IOP changes no longer parallel ICP changes and a pressure imbalance and subsequent rise in TLPG occur. On the other hand, the ONPG (i.e., IOP-ICP) linearly increases as ICP decreases, along with the TOPG (i.e., ONPG-ONSP). Thus, a relatively high IOP coincides with low ICP, meaning that, in this condition, IOP would press against the optic nerve head and affects the full length of the optic nerve in the optic canal. We propose that this imbalanced relationship between ICP and IOP may play a role in the development of glaucoma or optic neuropathy in patients with a lowered ICP. In other words, when ICP is too low, normally benign IOP levels can be harmful to the optic nerve.

Optic nerve anatomy can help explain the pressure dynamics that exist between ICP and IOP. The optic nerve passes through the intraocular, intra-orbital, and intracranial cavities, with each having their own pressure conditions (Figure 4).

A widely accepted possible cause of glaucomatous optic neuropathy is an increased TLPG, which causes optic nerve head damage. A stable TLPG value that remains within normal limits is important and necessary to maintain normal optic nerve function. In our dog study, TLPG remained relatively constant in the ICP-IOP dependent zone. This indi- cates that as long as IOP and ICP decline together, the pressure gradient on both sides of the lamina cribrosa remain relatively constant. However, when ICP and IOP become uncoupled (i.e., in the ICP-IOP independent zone), the pressures become out of balance and TLPG becomes unstable. As a result, average TLPG increased, unlike in the ICP-IOP dependent zone.

It is somewhat controversial whether or not TLPG changes caused by lower ICP have the same effect as IOP elevations (i.e., whether or not lamina cribrosa damage is the same). Hayreh et al. did not consider that a trans-lamina imbalance between IOP and ICP, caused by a lowered ICP, could result in glaucomatous optic neuropathy (Hayreh et al., 2009). This differs from views of other authors (Jonas et al., 2013; Siaudvytyte et al., 2015; Wostyn et al., 2011) who support the theory that lower ICP could cause glaucomatous optic neuropathy.

Both the eye-to-nerve contribution and the brain-to-nerve influence on optic neuropathy need to be considered to properly study these phenomena. As shown in Figure 4, the optic disk is not flat. Rather, it concaves towards the optic nerve longitudinal center. Therefore, the force generated by IOP exerts the greatest pressure on the central section of the optic nerve (i.e., the optic nerve center is more affected than more peripheral nerve tissue). In the intracranial cavity, the optic nerve is surrounded by CSF and is exposed to ICP. Thus, the ONPG is equal to IOP-ICP. Zhao et al. found that the ONPG plays an important role in optic nerve integrity and that increasing the ONPG can cause structural and functional damage to the optic nerve (Zhao et al., 2015). In our experiments, we observed an inverse relationship between ONPG and ICP (i.e., a decrease in ICP resulted in an increase in the ONPG). Figure 3 shows that the ONPG breakpoint is approximately $150 \%$. However, when the ICP decreases, ONPG can rise to as high as $300 \%$ (i.e., three times the baseline value). 


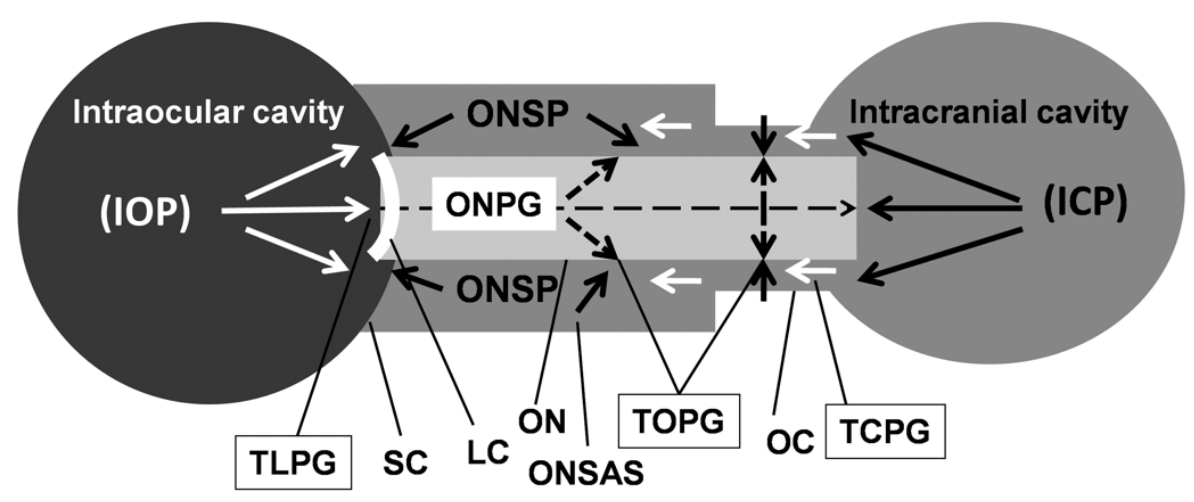

Figure 4 Pressures around the optic nerve (ON). From the retinal side, the ON head is located in the intraocular cavity and exposed to the intraocular pressure (IOP). In the intracranial cavity, the $\mathrm{ON}$ is connected with the optic chiasma and exposed to the intracranial pressure (ICP). Thus, there is a pressure gradient across the ON (ON pressure gradient (ONPG)) that can be calculated as IOP-ICP. In the orbital cavity, the ON is surrounded by the optic nerve subarachnoid space (SAS) and exposed to the optic nerve subarachnoid space pressure (ONSP). Thus, from the ON center to its periphery, there is a pressure gradient called the "trans-ON pressure gradient" (TOPG). Therefore, the ON is exposed to the longitudinal pressure gradient (ONPG) between the eye and the brain, and a lateral pressure gradient (TOPG) between the ONPG and ONSP. Therefore, the ON is exposed to a total of three pressure gradients, the two horizontal pressure gradients (trans-lamina cribrosa pressure gradient (TLPG) and trans-optic canal pressure gradient (TCPG)) and one lateral pressure gradient (TOPG). These gradients are regulated by the relationship between ICP and IOP, with ONSP mainly influenced by ICP through a functional fluid communication between the optic nerve SAS and the intracranial SAS via the optic canal (OC). Other pressures are determined by the correlation between ICP and IOP. LC, lamina cribrosa. SC, sclera canal.

In the orbital part of the optic nerve, the optic nerve sheath is rather elastic and can expand when the TOPG increases. In contrast, the optic nerve sheath in the optic canal is fixed on the optic canal and cannot expand or shrink. This implies that if the TOPG increases from the center to the periphery, the fiber bundles of the optic nerve will be compressed. It has been shown that the optic nerve is particularly susceptible to pressure changes and prone to deformation (Crawford Downs et al., 2011; Downs et al., 2008; Sigal et al., 2007). Even though these studies focused on the lamina cribrosa and the scleral canal, it is reasonable to assume that the optic canal is stiffer than the scleral canal. Thus, if the pressure gradient across the optic nerve increases, the optic nerve will be damaged in the optic canal because it cannot enlarge or deform to release the increasing pressure. As Table 3 shows, the TOPG was $16.4 \pm 13.7$ $\mathrm{mmH}_{2} \mathrm{O}$ at baseline, $38.8 \pm 15.1 \mathrm{mmH}_{2} \mathrm{O}$ in the ICP-IOP dependent zone, and $92.8 \pm 13.2 \mathrm{mmH}_{2} \mathrm{O}$ in the ICP-IOP independent zone. As ICP declines, the TOPG will gradually rise and push the optic nerve against the optic canal. This results in damage to the optic nerve, caused directly by the pressure gradient in it from the center to the periphery, and compression of the optic nerve SAS between the optic nerve and the optic canal. As a result, extra resistance for CSF movement from the cranial SAS to the optic nerve SAS develops and CSF circulation will be indirectly impaired. Thus, if the TOPG rises beyond its critical value, CSF flow from the cranial SAS into the optic nerve SAS will stop and compartment syndrome will develop, as proposed in our "dead pond theory" (Hou et al., 2016). This results in optic nerve damage directly from physical compression and/or indirectly from CSF circulatory disturbance, which would cause subsequent malnutrition and poisoning.
These considerations lead us to wonder how precisely ICP and IOP are related. Some investigators have argued that IOP is correlated with ICP (Lashutka et al., 2004; Li et al., 2012), but others argue that they are unrelated (Czarnik et al., 2009; Kirk et al., 2011). Still others have concluded that, although there is a correlation between ICP and IOP, IOP is a "poor predictor" of ICP (Golan et al., 2013; Spentzas et al., 2010; Yavin et al., 2014). Our study answers this question and shows that all authors are partially correct. Indeed, ICP and IOP are correlated, but only in the ICP-IOP dependent zone. However, in the ICP-IOP independent zone, ICP and IOP are not correlated.

The mechanism of ICP-IOP coupling in the ICP-IOP dependent zone is not well understood. Samuels et al. found that neurons within the dorsomedial hypothalamus (DMH) and surrounding perifornical area $(\mathrm{PeF})$ can influence both ICP and IOP (Samuels et al., 2012). They also showed that chemical stimulation of these structures can evoke substantial increases in IOP and ICP. Thus, certain brain areas simultaneously regulate both the ICP and IOP under normal conditions to maintain the balance between them. However, this regulation may be impaired when the DMH and/or PeF malfunction or when other factors dramatically affect ICP or IOP and overwhelm the regulatory power of the DMH and PeF. When this happens, ICP and IOP may become uncoupled and be independently regulated.

Though absolute IOP and ICP values are relevant, our data suggest that relative ICP and IOP changes that occur when ICP drops are more important. This is because the relative changes determine the different pressure gradients on the optic nerve (e.g., TLPG (IOP-ONSP), ONPG (IOPICP), TOPG (ONPG-ONSP), and TCPG (ICP-ONSP)). Thus, the combined influence of all pressures determine the 
physical pressure in the optic nerve and may inhibit or prevent CSF exchange, as proposed by the "dead pond theory" (Hou et al., 2016). When ICP decreases in the ICP-IOP dependent zone, these pressure gradients could be influenced by lowering ICP, but the changes may still be in an acceptable range when ICP and IOP are in balance. However, when ICP drops below a critical point, ICP and IOP become imbalanced and these pressures will dramatically change. When ICP and IOP exceed pathological levels, optic nerve structural and functional damage can occur. Our theory is compatible with the observations of Zhang et al. who observed that an acute ICP reduction could damage retinal ganglion cell axons (Zhang et al., 2015). This damage can be visualized using immunohistochemical analyses on axonal motor proteins, including kinesin $\mathrm{HC}$ and dynein IC.

Future studies are needed to determine if and how possible breakpoint elevation may be involved in glaucoma or optic neuropathy and how this parameter may contribute to optic nerve damage. It seems clear that a proper balance between ICP and IOP plays a critical role in determining pressures in the optic nerve chamber. Additionally, an imbalance may explain the development of some ophthalmological diseases, including glaucoma and optic neuropathy.

\section{MATERIALS AND METHODS}

\section{Animal preparation}

Eight mixed-breed beagles were studied in accordance with the Association for Research in Vision and Ophthalmology (ARVO) Statement for the Use of Animals in Ophthalmic and Vision Research. All applicable institutional and/or national guidelines for the care and use of animals were followed. Before implanting pressure probes, dogs were anesthetized with an intramuscular injection of ketamine $\mathrm{HCl}\left(20 \mathrm{mg} \mathrm{kg}^{-1}\right)$ and midazolam $\left(0.2 \mathrm{mg} \mathrm{kg}^{-1}\right)$. Additional ketamine $\left(10 \mathrm{mg} \mathrm{kg}{ }^{-1}\right)$ injections were administered as needed during examinations and surgery.

\section{Pressure transducers and probes}

Pressures in the four examined cavities were simultaneously measured using hydraulic pressure transducers that had been custom made for our laboratory by the Biological Mechanics Laboratory at Beijing University of Aeronautics \& Astronautics. A single transducer was implanted into the left ventricle, optic nerve SAS of the left eye, lumbar cistern, and anterior chamber of the left eye. The devices consisted of a computer, an amplifying device, pressure sensors, and probes. The absolute pressure measurement error for each transducer was $\pm 1.0 \mathrm{mmH}_{2} \mathrm{O}$. Each manometer was connected to a pressure sensor via a three-way union and connected with the amplifier device. Pressure signals were converted into electrical signals and recorded in real-time so that ICP, LCP, ONSP, and IOP could all be recorded simultaneously. All tubing was filled with normal saline (NS) and any air in the tubes was removed. The three-way union made it possible to wash the device, which was linked with the pressure measurement system. This set-up allowed us to flush the system without removing the probe so that any tissue that had plugged the front-end of the needle during insertion could be removed.

\section{Implanting pressure transducers}

Probes were implanted after dogs were properly anaesthetized (see above) and suspended in the prone position. The front legs hung down through a fenestrated sheet, but the rest of the body was supported by a sling. The head position was fixed with a maxilla bite clamp that was connected to the operating table. A skin incision was made over the 4th lumbar vertebra and a laminotomy was performed using blunt dissection to cannulate the lumbar cistern. Electrocoagulation and electrotomy were used to avoid excessive bleeding and vertebral bone was removed to access the dura mater. A catheter connected to a pressure sensor was then implanted into the lumbar cistern.

The IOP was measured with a 25 -gauge cannula connected to a pressure transducer. The cannula was inserted into the anterior chamber approximately $2 \mathrm{~mm}$ temporal to the limbus and was positioned close to the center of the pupil.

The ICP was measured through a burr hole on one side that was drilled $1 \mathrm{~cm}$ lateral to the sagittal midline, halfway between the occipital prominence and a line between both lateral canthi. The dura was then incised and the lateral ventricle was punctured using a Frazier's ventricular needle. Next, a catheter was inserted into the frontal horn of the lateral ventricle on the other side. The left catheter was connected to a pressure sensor and the right catheter served to automatically subtract out pressure changes resulting from possible CSF volume alterations.

Lastly, a lateral orbitotomy was performed to expose the left retrobulbar optic nerve sheath. Extreme care was taken when puncturing the optic nerve sheath with a sharp 28-guage needle to gain access to the optic nerve SAS. Accurate puncture was performed by first connecting the pressure transducer so that the zero-pressure-baseline could be measured. We then slowly punctured the optic nerve sheath while simultaneously monitoring pressure. As the needle punctured the dura, a successful probe entry was clearly indicated by a sharp real-time pressure rise. Pressure was then monitored for at least $5 \mathrm{~min}$ to assure that CSF baseline pressure was stable. The needle and pressure probe were secured into place using a biological glue (ECglue, Guangzhou Baiyun Medical Glue Company, China), which was also used to close the puncture site to prevent CSF leakage.

\section{Data processing}

Calculations of pressures were performed with MATLAB (MathWorks Inc., USA). To avoid interpretation errors, all 
pressure recordings were manually inspected for any unusual artifacts and/or fluctuations. For each $1.0 \mathrm{~s}$ period, pressures were calculated as the mean of unfiltered pressure; using $10^{\text {th }}$ order Butterworth zero phase filters we removed slow respirator waves (high pass cut-off frequency $0.5 \mathrm{~Hz}$ ).

Pressures were calculated by first measuring the distances between puncture points and the operating table. Probes were then connected to the pressure monitoring system so that the hydrostatic zero-pressure-baseline could be determined. Pressure probes for each puncture point were then placed to collect baseline values for ICP, LCP, ONSP, and IOP. Each pressure was recorded for at least $5 \mathrm{~min}$. Pressure values were determined by subtracting the starting point (zero-pressure-baseline) values from real-time values. All pressure probes were continuously monitored during the 5 min baseline and 3-5 min shunting event recordings. To achieve a reliable and stable pulsatility curve, ICP values were divided into $10 \mathrm{mmH}_{2} \mathrm{O}$ intervals. Intervals with fewer than three values were discarded to minimize the influence of noise. The median was used to reduce the influence of single outlier values.

The relative pressure coefficient was calculated as the slope of a linear regression of ICP, LCP, ONSP, and IOP. The TLPG was essentially constant when ICP and IOP were synchronized (see Results) and was used to determine the "breakpoint" (Figure 2A and B). This was defined as the value at which the transition between the "ICP-IOP dependent zone" (ICP and IOP values dropped in parallel) and the "ICP-IOP independent zone" (IOP decrease no longer accompanied by an ICP decrease) occurred.

\section{Statistics}

Mean IOP, ICP, LCP, and ONSP values were compared using paired $t$-tests. All statistical analyses were conducted using Origin Statistics (V.9.1; OriginLab Corporation, USA). Statistical significance was defined as $P<0.05$.

Compliance and ethics All applicable institutional and/or national guidelines for the care and use of animals were followed. The author(s) declare that they have no conflict of interest.

Acknowledgements This study was supported by the National Natural Science Foundation of China (81271005, 81300767), Beijing Natural Science Foundation (7122038), and two separate donations by the China Health and Medical Development Foundation. B.A.S. was supported by the $B M B F$ network ERA-net Neuron "Restoration of Vision after Stroke (REVIS)" (BMBF 01EW1210), and by the "Hai-ju" Beijing Overseas Talents Program. Ruowu Hou was supported by the Beijing Tongren Hospital Foundation (2015-YJJ-GGL-013).

Bach-Holm, D., Kessing, S.V., Mogensen, U., Forman, J.L., Andersen, P.K., and Kessing, L.V. (2012). Normal tension glaucoma and Alzheimer disease: comorbidity? Acta Ophthalmol 90, 683-685.

Berdahl, J.P., Allingham, R.R., and Johnson, D.H. (2008a). Cerebrospinal fluid pressure is decreased in primary open-angle glaucoma. Ophthalmology $115,763-768$.
Berdahl, J.P., Fautsch, M.P., Stinnett, S.S., and Allingham, R.R. (2008b). Intracranial pressure in primary open angle glaucoma, normal tension glaucoma, and ocular hypertension: a case-control study. Invest Ophthalmol Vis Sci 49, 5412-5418.

Crawford Downs, J., Roberts, M.D., and Sigal, I.A. (2011). Glaucomatous cupping of the lamina cribrosa: a review of the evidence for active progressive remodeling as a mechanism. Exp Eye Res 93, 133-140.

Czarnik, T., Gawda, R., Kolodziej, W., Latka, D., Sznajd-Weron, K., and Weron, R. (2009). Associations between intracranial pressure, intraocular pressure and mean arterial pressure in patients with traumatic and non-traumatic brain injuries. Injury 40, 33-39.

Downs, J.C., Roberts, M.D., and Burgoyne, C.F. (2008). Mechanical environment of the optic nerve head in glaucoma. Optom Vis Sci 85, $425-435$.

Hou, R., Zhang, Z., Yang, D., Wang, H., Chen, W., Li, Z., Sang, J., Liu, S., Cao, Y., Xie, X., Ren, R., Zhang, Y., Sabel, B.A., Wang, N. (2016). Pressure conditions in the optic nerve chamber: correlation of intracranial pressure (ICP) and optic nerve subarachnoid space pressure (ONSP). Brain Res doi: 10.1016/j.brainres.2016.01.011.

Golan, S., Kurtz, S., Mezad-Koursh, D., Waisbourd, M., Kesler, A., and Halpern, P. (2013). Poor correlation between intracranial pressure and intraocular pressure by hand-held tonometry. Clin Ophthalmol 7, 1083-1087.

Hayreh, S.S. (2009). Cerebrospinal fluid pressure and glaucomatous optic disc cupping. Graefes Arch Clin Exp Ophthalmol 247, 721-724.

Jonas, J.B., Yang, D., and Wang, N. (2013). Intracranial pressure and glaucoma. J Glaucoma 22 Suppl 5, S13-S14.

Kirk, T., Jones, K., Miller, S., and Corbett, J. (2011). Measurement of intraocular and intracranial pressure: is there a relationship? Ann Neurol 70, 323-326.

Lashutka, M.K., Chandra, A., Murray, H.N., Phillips, G.S., and Hiestand, B.C. (2004). The relationship of intraocular pressure to intracranial pressure. Ann Emerg Med 43, 585-591.

Li, Z., Yang, Y., Lu, Y., Liu, D., Xu, E., Jia, J., Yang, D., Zhang, X., Yang, H., Wang, N., and Ma, D. (2012). Intraocular pressure vs intracranial pressure in disease conditions: a prospective cohort study (Beijing iCOP study). BMC Neurol 12, 66.

Mader, T.H., Gibson, C.R., Pass, A.F., Kramer, L.A., Lee, A.G., Fogarty, J., Tarver, W.J., Dervay, J.P., Hamilton, D.R., Sargsyan, A., Phillips, J.L., Tran, D., Lipsky, W., Choi, J., Stern, C., Kuyumjian, R., and Polk, J.D. (2011). Optic disc edema, globe flattening, choroidal folds, and hyperopic shifts observed in astronauts after long-duration space flight. Ophthalmology 118, 2058-2069.

Ren, R., Jonas, J.B., Tian, G., Zhen, Y., Ma, K., Li, S., Wang, H., Li, B., Zhang, X., and Wang, N. (2010). Cerebrospinal fluid pressure in glaucoma: a prospective study. Ophthalmology 117, 259-266.

Samuels, B.C., Hammes, N.M., Johnson, P.L., Shekhar, A., McKinnon, S.J., and Allingham, R.R. (2012). Dorsomedial/Perifornical hypothalamic stimulation increases intraocular pressure, intracranial pressure, and the translaminar pressure gradient. Invest Ophthalmol Vis Sci 53, 7328-7335.

Siaudvytyte, L., Januleviciene, I., Ragauskas, A., Bartusis, L., Siesky, B., and Harris, A. (2015). Update in intracranial pressure evaluation methods and translaminar pressure gradient role in glaucoma. Acta Ophthalmol 93, 9-15.

Sigal, I.A., Flanagan, J.G., Tertinegg, I., and Ethier, C.R. (2007). Predicted extension, compression and shearing of optic nerve head tissues. Exp Eye Res 85, 312-322.

Spentzas, T., Henricksen, J., Patters, A.B., and Chaum, E. (2010). Correlation of intraocular pressure with intracranial pressure in children with severe head injuries. Pediatr Crit Care Med 11, 593-598.

Woo, S.J., Kim, T.W., and Chung, K.Y. (2012). Intraocular pressure elevation during space flight. J Glaucoma 21, 349.

Wostyn, P., Audenaert, K., and De Deyn, P.P. (2008). Alzheimer's disease-related changes in diseases characterized by elevation of intracranial or intraocular pressure. Clin Neurol Neurosurg 110, 101-109.

Wostyn, P., Audenaert, K., and De Deyn, P.P. (2010). Alzheimer's disease: cerebral glaucoma? Med Hypotheses 74, 973-977. 
Wostyn, P., De Groot, V., Audenaert, K., and De Deyn, P.P. (2011). Are intracranial pressure fluctuations important in glaucoma? Med Hypotheses 77, 598-600.

Yang, D., Fu, J., Hou, R., Liu, K., Jonas, J.B., Wang, H., Chen, W., Li, Z., Sang, J., Zhang, Z., Liu, S., Cao, Y., Xie, X., Ren, R., Lu, Q., Weinreb, R.N., and Wang, N. (2014). Optic neuropathy induced by experimentally reduced cerebrospinal fluid pressure in monkeys. Invest Ophthalmol Vis Sci 55, 3067-3073.

Yavin, D., Luu, J., James, M.T., Roberts, D.J., Sutherland, G.R., Jette, N., and Wiebe, S. (2014). Diagnostic accuracy of intraocular pressure measurement for the detection of raised intracranial pressure: meta-analysis: a systematic review. J Neurosurg 121, 680-687.

Zhang, Z., Wu, S., Jonas, J.B., Zhang, J., Liu, K., Lu, Q., and Wang, N. (2015). Dynein, kinesin and morphological changes in optic nerve axons in a rat model with cerebrospinal fluid pressure reduction: the Beijing Intracranial and Intraocular Pressure (iCOP) study. Acta Ophthalmol doi: 10.1111/aos.12768.

Zhao, D., He, Z., Vingrys, A.J., Bui, B.V., and Nguyen, C.T. (2015). The effect of intraocular and intracranial pressure on retinal structure and function in rats. Physiol Rep doi: 10.14814/phy2.12507.

Open Access This article is distributed under the terms of the Creative Commons Attribution License which permits any use, distribution, and reproduction in any medium, provided the original author(s) and source are credited. 